\title{
Differential Expression of ANXA2 in Eutopic and Ectopic Endometrium
}

\author{
HAIYAN YU, QINGLING LU ${ }^{1}$, YITING WANG ${ }^{1}, X . M A$, BO LI ${ }^{1}$ AND LINA YANG ${ }^{1 *}$ \\ Health Management Center, ${ }^{1}$ Department of Gynecology, Heilongjiang Provincial Hospital, Harbin City, 150036, Heilongjiang \\ Province, China
}

Yu et al.: Differential Expression of ANXA2 in Eutopic and Ectopic Endometrium

\begin{abstract}
The purpose of this study was to compare the differences in expression of annexin A2 between eutopic and ectopic endometria and to explore the pathogenesis of adenomyosis. In this study, 22 adenomyosis patients who underwent uterine laparotomy in Heilongjiang Provincial Hospital from April 2016 to October 2018 were enrolled as the observation group. The eutopic and ectopic endometria were used for the experiment. In addition, 22 uterine fibroids patients with normal endometrium who underwent open hysterectomy were selected as the control group. The levels of annexin $\mathrm{A} 2$ in endometrial tissue and serum were compared between the two groups. The results showed that the annexin $\mathrm{A} 2$ level in normal endometrial tissue was significantly lower than that in the eutopic and ectopic endometrial tissue and the annexin A2 level in the ectopic endometrium was significantly higher than that in the eutopic endometrium $(p<0.05)$. The annexin A2 level in the serum of the observation group was significantly $(p<0.05)$ higher than that of the control group. Clinical monitoring of changes in annexin $\mathrm{A} 2$ level could be useful as auxiliary diagnostic marker to start treatment of adenomyosis, so as to project prognostic changes more accurately. However, the results of this study still need to be verified with multi-center samples.
\end{abstract}

Key words: Annexin A2, eutopic endometrium, ectopic endometrium, expression difference, adenomyosis

Adenomyosis (AM) mainly refers to a type of disease in which benign infiltration and growth occur constantly in the glands or interstitium of the endometrium towards the muscular layer, causing symptoms such as dysmenorrhea or excessive menstruation ${ }^{[1]}$. This is an estrogen-dependent disease that can be pathologically benign. However, some biological behaviors, such as implantation, infiltration and recurrence of malignant tumors have a great impact on patients' quality of life $\mathrm{e}^{[2-4]}$. Detection of serum CA125 is helpful for diagnosis, but final diagnosis still requires surgery and pathology.
Due to the lack of reliable diagnostic indicators, it is inevitable to misdiagnose or miss the diagnosis. Therefore, it is of great significance to search for a diagnostic marker. In addition, moderate and severe dysmenorrhea seriously affects the physical and mental health and quality of life of women of childbearing age but the relevant mechanism is still not very clear and the root cause urgently needs to be investigated.

Annexin A2, also known as Annexin II, AXII and ANXA2. ANXA2 is more commonly expressed in human endothelial cells, neutrophils, bone marrow 
cells, brain cells and some tumor cells. It is highly expressed in cells with high proliferation and differentiation ability, and is expressed in cells with low differentiation. About 1/3rd of ANXA2 is produced by endothelial cells, which play a very important role in the cells in and out of the body ${ }^{[5]}$. Within the cell, it is responsible for cell proliferation, differentiation, apoptosis, DNA synthesis, RNA binding, mRNA transport, cytoskeletal remodeling and stabilization, biofilm formation, vesicle trafficking. Outside the cell, it is responsible for osteoblast formation and bone resorption, channel formation of $\mathrm{Ca}^{2+}$, opening and regulation of ion flux frequency, signal transduction, immunoglobulin transport, cell metastasis and invasion, epithelial mesenchymal transition (EMT), fibrinolysis, and the formation of new blood vessels. And in many diseases, such as human immunodeficiency virus (HIV), hepatitis B, hepatitis C, diabetic nephropathy, thyroid disease, sepsis, endometriosis, melanoma, psoriasis, keloid, acute young granulocyte leukemia, lymphoma, multiple myeloma, acute promyelocytic leukemia, lupus nephritis and malignant tumor, it also plays a very important role. In addition, it can serve as a specific anchor protein for bacteria and viruses and is also involved in the regulation of redox reactions in cells $^{[6-8]}$.

It was found that estrogen in the female could significantly regulate the expression of ANXA2, which could affect the incidence of AM by means of endometrial metastasis and angiogenesis. In recent years, it was reported that the EMT is the initiator of the pathogenesis of $\mathrm{AM}^{[9]}$. Studies on in vitro $\mathrm{AM}$ model confirmed that estrogen can significantly up-regulate ANXA2 and induce the occurrence of EMT, suggesting that ANXA2 may be related to the occurrence of AM. Some studies have also confirmed that ANXA2 plays an active role in AM development by endometrial metastasis and angiogenesis. In this study, the expression of ANXA2 in AM tissues and serum of the eutopic endometrium and ectopic endometrium was detected by immunohistochemical S-P assay and enzyme-linked immunosorbent assay (ELISA) to explore its mechanism of action in the occurrence and development of AM, so as to provide a reliable theoretical basis for the clinical diagnosis and treatment of AM and the development and application of relevant markers.

From April 2016 to October 2018, 22 AM patients, aged 31-46 y, with an average age of $42.55 \pm 4.82 \mathrm{y}$, who underwent laparoscopic hysterectomy in the
Heilongjiang Provincial Hospital were enrolled as the observation group. The eutopic and ectopic endometria were used for experiments. Thirty two preoperative patients with synchronous uterine fibroids were selected, the patients were aged 33-47 y, with an average age of $43.64 \pm 5.65 \mathrm{y}$. All of them were confirmed to have uterine fibroids and normal endometrium on postoperative pathology.

During the same period, 22 cases of normal endometrium in patients with uterine fibroids undergoing laparoscopic hysterectomy in the Heilongjiang Provincial Hospital were selected, who were aged from 32 to $46 \mathrm{y}$, with an average age of $42.60 \pm 4.74 \mathrm{y}$ as the control group. Thirtytwo preoperative serums of patients with synchronous uterine fibroids were selected. The patients were 33 to $48 \mathrm{y}$, with an average of $44.02 \pm 5.95 \mathrm{y}$. All of them were confirmed to have uterine fibroids and normal endometrium by postoperative pathology.

All specimens were strictly screened as premenopausal women with no endometrial atypical hyperplasia. The patient should have no other endocrine, immune and metabolic diseases, malignant tumors, and did not receive any hormone or immunotherapy within 3 mo before surgery. This study was approved by the medical ethics committee of the Heilongjiang Provincial Hospital, all patients were informed and gave consent to the content of this study and all pathological sections were reviewed by the pathologist.

In this study, the tissue was excised by paraffin embedding surgery and stained with a $4 \mu \mathrm{m}$ thick section. All specimens were operated according to kit instructions under the same conditions, and PBS was used as negative control instead of primary antibody. The specific operation process is shown in fig. 1 .

The specific reaction of antigen and antibody is used to connect the substance to enzyme and then the color reaction is produced between enzyme and substrate for quantitative determination. The subject to be measured may be an antibody or an antigen. The method of labeling with fluorescein is called immunofluorescence and the commonly used luciferin is fluorescein isothiocyanate, rhodamine and so on. The commonly used enzyme is horseradish peroxidase. The enzyme reacts with the substrate to form an opaque deposit, which shows the position of the presence of the antigen. In this study, $3 \mathrm{ml}$ of venous blood was taken on an empty stomach in the morning before the open hysterectomy and the serum was separated and stored at $-80^{\circ}$. The enzyme labeling instrument was Bio-Rad680 product (Bio-Rad, 


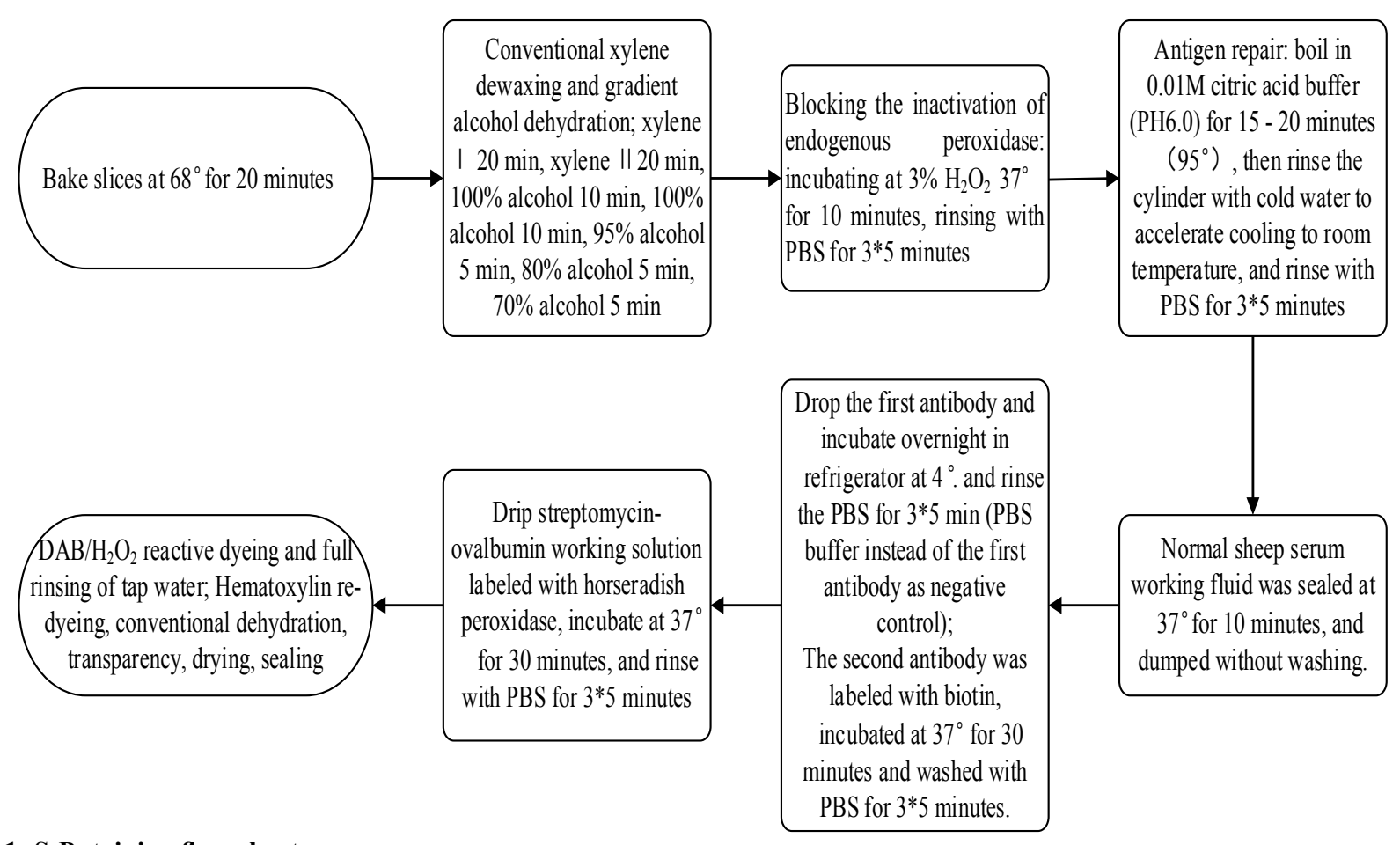

Fig. 1: S-P staining flow chart

USA). The procedure described in the kit was followed strictly during testing.

The data were processed by SPSS20.0 statistical software and the measurement data were expressed as mean \pm standard deviation. $t$ test was used to compare serum concentrations between groups, and $p<0.05$ was considered statistically significant.

Studies have found that the promotion of endometrial tissue growth, distant metastasis and angiogenesis in $\mathrm{AM}$ patients is achieved through the mechanism of ANXA2-induced EMT. The basic structure of ANXA2 contains 339 amino acids, consisting of a $3 \mathrm{kD} \mathrm{N}$-terminus and a $33 \mathrm{kD}$ C-terminal domain. As a calcium-binding cytoskeleton protein, it is involved in angiogenesis, proliferation, apoptosis, transduction of calcium signal and cell growth regulation. The mechanism of its induction of EMT in AM may be that it is hydrolyzed into fibrinolytic enzyme after binding to fibrinolytic progenitor and fibrinolytic enzyme has the function of degrading extracellular matrix and basement membrane of peripheral blood vessels. It can also bind to adhesion molecules on the surface of cell, so as to change the adhesion between cells and between cells and matrix, enhance the antiapoptotic ability and motor ability of cells and develop EMT. The levels of ANXA2 in the endometrial tissues of the two groups were compared. It was found that the level of ANXA2 in the normal endometrial tissue of the control group was significantly lower than that in the ectopic and the eutopic endometrium tissue in the observation group $(p<0.05)$ and the level of ANXA2 in the ectopic endometrial tissue was significantly higher than that in the eutopic endometrial tissue $(p<0.05)$, as shown in Table 1 and fig. 2.

ANXA2 is a calcium-mediated phospholipid-binding protein, a member of the membrane protein family, widely distributed in the nucleus, cytoplasm and cytoplasmic membrane surface. In cells, ANXA2 is involved in a series of important life processes including membrane formation, membrane transport, endocytosis, exocytosis, cell proliferation, signal transduction, differentiation and apoptosis. In patients with elevated $\mathrm{AM}$, estrogen might increase cyclooxygenase 2 by upregulating ANXA2, thereby increasing the production of prostaglandin E2 and promoting the production and exacerbation of dysmenorrhea. EMT is also closely related to the high estrogen environment, and estrogeninduced EMT is one of the important mechanisms of AM development, in which ANXA2 may play a key role. In this study, the ANXA2 level in serum in the observation group was significantly higher than that in the control group, and the difference between the two groups was statistically significant $(p<0.05)$, as shown in Table 2. This suggested that the level of ANXA2 in serum was also significantly increased in patients with AM, which may be related to the fact that the level of ANXA2 was up-regulated by the disorder of estrogen metabolism in patients. 
TABLE 1: COMPARISON OF ANXA2 LEVELS IN ENDOMETRIAL TISSUE BETWEEN THE TWO GROUPS

\begin{tabular}{llcc}
\hline Group & & Cases & ANXA2 \\
\hline Control group & Normal endometrium & 22 & $3.80 \pm 2.62$ \\
Observation & Eutopic endometrium & 22 & $7.88 \pm 2.04$ \\
group & Ectopic endometrium & 22 & $8.42 \pm 1.98$ \\
\hline
\end{tabular}

Mean \pm standard deviation, $\mathrm{ng} / \mathrm{ml}$

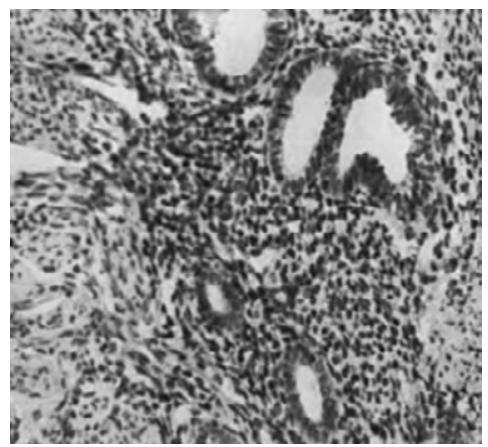

(a)

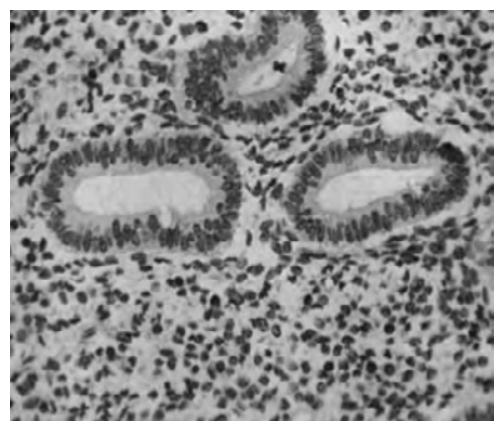

(b)

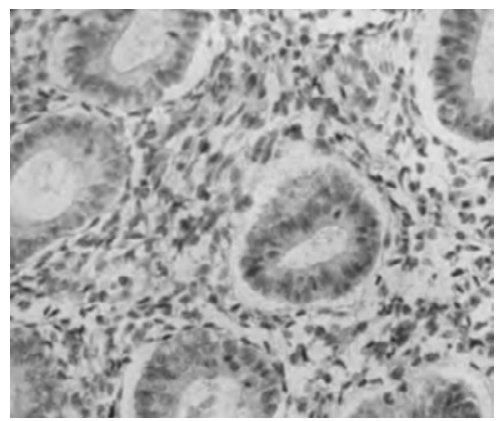

(c)

Fig. 2: Expression of ANXA2 in the intimal tissue of two groups of patients

(a) Expression of ANXA2 in ectopic endometrium of patients with adenomyosis. (b) Expression of ANXA2 in the eutopic endometrium of patients with adenomyosis. (c) Expression of ANXA2 in normal endometrium

ANXA2 was found to be abnormally expressed in cervical cancer, ovarian cancer, choriocarcinoma and other gynecological malignancies. AM also had similar biological behavior to malignant tumors. It was speculated that ANXA2 may be abnormally expressed in human AM and promote the occurrence and development of AM, but relevant studies have not been reported. The level of ANXA2 in peripheral blood of patients with uterine fibroids was significantly lower than that of patients with AM $(\mathrm{p}<0.05)$. This indicated that the level of ANXA2 in peripheral blood had a certain differentiation effect between AM and uterine fibroids, suggesting that ANXA2 may be a new marker for AM diagnosis. Therefore, changes in the level of ANXA2 need to be monitored to assist in the diagnosis and treatment of patients with AM.

AM mostly occurs in middle-aged women and its main clinical symptoms are secondary dysmenorrhea and increased menstrual volume, as well as infertility. Currently, hysterectomy is the most effective treatment option. However, this surgical method is often hard to accept for some patients who have fertility needs or desire to retain the uterus and the most accurate means of diagnosis nowadays is postoperative histopathological examination. Therefore, it is of great significance to search for an early biomarker to evaluate AM. It has been reported that the incidence of AM may be related to EMT and ANXA2 could induce this transformation mechanism. ANXA2, as the co-receptor of tissue plasminogen activator and plasminogen, can be produced by plasminogen mediated and catalyzed by cell surface layer. In endothelial cells, mononuclear or macrophages, and some tumor cells, it can participate in biological membrane and ion channels, cytoskeleton, cell proliferation, differentiation, and other processes, which are associated with endometrial lesions.

The results of this study showed that the level of ANXA2 in the normal endometrial tissue was significantly lower than that in the eutopic and ectopic endometria $(\mathrm{p}<0.05)$, suggesting that the level of ANXA2 in the endometrial tissue of patients with AM was significantly increased. This could be related to the involvement of ANXA2 in the pathogenesis and progression of AM. As a calciumbinding cytoskeletal protein, ANXA2 can regulate the growth, proliferation, and apoptosis of cells or blood

TABLE 2: COMPARISON OF ANXA2 LEVELS IN SERUM OF TWO GROUPS OF PATIENTS

\begin{tabular}{lcc}
\hline Group & Cases & ANXA2 \\
\hline Control group & 22 & $7.78 \pm 2.11$ \\
Observation group & 22 & $8.28 \pm 1.72$ \\
$\mathrm{t}$ & - & 2.562 \\
$\mathrm{P}$ & - & $<0.05$ \\
Adenomyosis group & 32 & $3.14 \pm 0.52$ \\
Fibroid group & 32 & $1.04 \pm 0.28$ \\
$\mathrm{t}$ & - & 20.774 \\
$\mathrm{P}$ & - & $<0.05$ \\
\hline
\end{tabular}

Mean \pm standard deviation, $\mathrm{ng} / \mathrm{ml}$

Special Issue 2, 2020 
vessels and the conduction of calcium signals and can be hydrolyzed into fibrinolytic enzyme by combining with plasminogen, resulting in the degradation of extracellular matrix and vascular basement membrane. In addition, it can be combined with adhesion molecules on the cell surface, leading to changes in adhesion between cells and the matrix, strengthening the antiapoptosis and motor ability of cells themselves, and eventually causing AM.

In this study, for the first time, ANXA2 in serum of patients with AM was determined using ELISA before surgery and it was significantly higher than that in patients with uterine fibroids, suggesting that ANXA2 could serve as a new marker for AM diagnosis. Therefore, changes in the level of ANXA2 should be monitored clinically to assist the diagnosis and treatment of patients with AM, so as to grasp their conditions and predict prognosis changes more accurately. However, this study needs to be verified by multi-center samples.

\section{REFERENCES}

1. Liu LX, Wu YG, Zheng J. Increased annexin A2 and decreased $\beta$-catenin in adenomyosis contribute to adenomyosisassociated dysmenorrhea. Histol Histopathol 2017;32:11906.

2. Jiang C, Liu C, Guo J. The Expression of Toll-like receptors in eutopic and ectopic endometrium and its implication in the inflammatory pathogenesis of adenomyosis. Sci Rep 2017;7(1):7365.

3. Arlier S, Arıc1 A. Tumor necrosis factor alfa and interleukin 1 alfa induced phosphorylation and degradation of inhibitory kappa B alpha are regulated by estradiol in endometrial cells. Turk J Obstet Gynecol 2018;15(1):50-9.

4. Zou Y, Liu FY, Wang LQ. Downregulation of DNA methyltransferase 3 alpha promotes cell proliferation and invasion of ectopic endometrial stromal cells in adenomyosis. Gene 2017;604:41-7.

5. Lin X, Dai Y, Xu W. Hypoxia Promotes Ectopic Adhesion Ability of Endometrial Stromal Cells via TGF- $\beta 1 / \mathrm{Smad}$ Signalling in Endometriosis. J Endocrinol 2018;159(4):163041.

6. Ji F, Yang X, He Y. Aberrant endometrial DNA methylome of homeobox A10 and catechol-O-methyltransferase in endometriosis. J Assist Reprod Genet 2017;34(3):409-15.

7. Patel BG, Rudnicki M, Yu J, Shu Y, Taylor RN. Progesterone resistance in endometriosis: origins, consequences and interventions. Acta Obstet Gynecol Scand 2017;96(6):623-32.

8. Zhao L, Gu C, Ye M, Zhang Z, Li L, Fan W, et al. Integration analysis of microRNA and mRNA paired expression profiling identifies deregulated microRNA-transcription factor-gene regulatory networks in ovarian endometriosis. Reprod Biol Endocrinol 2018;16(1):4.

9. Guo SW. Fibrogenesis resulting from cyclic bleeding: the Holy Grail of the natural history of ectopic endometrium. Hum Reprod 2018;33(3):353-6.

This is an open access article distributed under the terms of the Creative Commons Attribution-NonCommercial-ShareAlike 3.0 License, which allows others to remix, tweak, and build upon the work non-commercially, as long as the author is credited and the new creations are licensed under the identical terms

This article was originally published in a special issue: Special issue on "Drug Development and Human Health in China"

Indian J Pharm Sci 2020:82(1)spl issue2;35-39 